\title{
Note de recherche sur le comput du temps chez les Bijogo de Buhaque
}

\section{Christine Henry}

\section{(2) OpenEdition \\ 1 Journals}

Édition électronique

URL : http://journals.openedition.org/span/604

DOI : $10.4000 /$ span. 604

ISSN : 2268-1558

Éditeur

École pratique des hautes études. Sciences humaines

\section{Édition imprimée}

Date de publication : 1 septembre 1986

Pagination : 143-147

ISSN : 0294-7080

\section{Référence électronique}

Christine Henry, "Note de recherche sur le comput du temps chez les Bijogo de Buhaque », Systèmes

de pensée en Afrique noire [En ligne], 7 | 1986, mis en ligne le 05 juin 2013, consulté le 06 mai 2019

URL : http://journals.openedition.org/span/604 ; DOI : 10.4000/span.604 


\title{
NOTE DE RECHERCHE SUR LE COMPUT DU TEMPS CHEZ LES BIJOGO DE BUBAQUE:
}

\author{
par \\ Christine Henry
}

Les Bijogo forment un groupe d'environ 15000 personnes occupant une dizaine d'îles de $I^{\prime}$ archipel des Bijagos (Guinée-Bissau). Ils vivent dans de petits villages construits dans des clairières et s'adonnent principalement à la monoculture du riz. Matrilinéaires, les Bijogo pratiquent l'alliance, généralement virilocale, sans compensation matrimoniale, dont le trait dominant èst l'indépendance de la femme dans ses choix et ses mouvements. L'organisation sociale dont l'unité de base est le village est caractêrisêe par l'importance des classes d'âge. Le principe de séniorité prévaut sur tout autre principe de subordination et $n$ 'écarte pas les femmes qui peuvent accéder à d'importantes charges $r i-$ tuelles. Les Bijogo parlent une langue à classes nominales du groupe Ouest-Atlantique qui n'a pas été étudiée jusqu'à présent (1).

\section{La notion de temps}

Dans le champ sémantique du temps, I'un des termes les plus importants de la langue bijogo est celui de kapótó (2) (moment, période, saison). On 1 'emploie dans des constructions de ce genre:

-- nous partirons le matin :

$$
\begin{aligned}
& \text { ate tibado an kapótó kari kankooya } \\
& \text { nous partirons dans temps đu matin }
\end{aligned}
$$

-- j'ai acheté cette bicyclette à l'époque du défrichage :

$$
\text { ñeresak jéc-jéc ena an kapótó ka kadém }
$$

j'ai acheté bicyclette cette au moment du défrichage

-- je n'ai pas eu le temps de finir ce travail :

ñakansaki edakatu ram kapótó kankonoken

je n'ai pas achevé travail parce que temps court trop

Le comput du temps utilise un double système solaire et lunaire.

Systèmes de pensée, Cahier 7, 1984 


\section{L'année}

Une saison humide amenobe et une saison sèche annine constituent une année nekena. Le dêbut de 1 'année coľncide avec le début de la saison humide. Les Bijogo ne nomment que les lunaisons de la saison humide et disent que leur année est de sept lunes (etaaku). Le fait qi'il y ait plus de sept lunaisons dans la somme d'une saison humide et d'une saison sèche ne semble pas faire problème. On dit toujours qu'une année est faite de deux saisons et de sept lunes. S'agit-il d'un phénomène sémantique comparable à celui du mot jour en français qui peut désigner à la fois une unité de temps de 24 heures et les seules heures éclairées de la journée ? Et de quoi serait révélatrice 1 'association de ces traits sémantiques?

D. Liberski fait état, chez les Kasena, de phénomènes similaires et suggère que "le jour, le mois, I'annêe se structurent sur le modèle de l'alternance du jour (comptê) et de la nuit (non comptêe)" (3). Contrairement aux Kasena, les Bijogo ne font pas eux-mêmes état d'un tel paradigme quand ils parlent du décompte des lunaisons dans 1 'annêe. Je n'oserai donc pas aller jusqu'à dire que ce modèle prêside également à leur conception du temps. Néanmoins, il semble que la représentation du temps chez les Bijogo ne soit ni linéaire ni cyclique, mais, comme le dit Leach (4), "pendulaire", fondée sur un vaet-vient entre deux pôles, constituée "d'opposês qui se répètent" : humidité/sêcheresse, jour/nuit, lune croissante/lune décroissante; "une discontinuité de contrastes répétés".

Un autre indice d'une conception non cyclique du temps se donne peut-être à lire dans le fait qu'il n'existe pas de division du temps supérieure à une année alors que les rituels les plus importants dans cette société sont les rites initiatiques masculins et féminins qui ont lieu régulièrement (5) selon les îles tous les six à dix ans. Cette période de temps ne reçoit pas de nom particulier bien que certains vieillards à qui on demande leur âge répondent qu'ils ont "vu beaucoup d'initiations".

Pour se situer dans la succession des années, on parlera de 1'année où nous sommes : nekena enna (cette annēe), de l'année dernière : nekena no naando ( 1 'année qui est passée), de l'année prochaine : nekena ni badano ( 1 'année qui va venir).

Pour parler de temps plus anciens, on rappelera les événements qui ont marqué 1 'époque. Les Bijogo ne connaissent pas leur âge en nombre d'années et, quand on leur pose la question, ils répondent en se situant relativement à quelqu'un d'autre : "j'ai le même âge que $X^{\prime \prime}$. Les campagnes de vaccination effectuées par les services de santê demandent une connaissance plus précise de l'âge des enfants. Le repérage sera alors effectué par rapport à l'emplacement des champs cultivés I'année de leur naissance (les Bijogo pratiquent une culture itinérante sur brûlis, rotative ou non selon les illes). Cette opêration n'est pas spontanée et donne lieu à de nombreuses réflexions et discussions entre mères comme me le signalait le médecin de Bubaque.

La saison humide

Le terme etaaku dẻsigne à la fois la lune et une lunaison. Lorsque les changements dans les conditions climatiques et dans l'aspect de la flore annoncent l'arrivée de la pluie commence le décompte des 
Iunaisons.

La première est nommée kobeetu (moitié) car c'est une période à moitié sèche et à moitié humide.

La seconde era kobeetu (après moitiê). Celle qui vient après la lunaison dénommée moitîe.

La troisième eran kaduubi (le temps de sarcler).

La quatrième topooji (recherche). C'est l'époque où le riz commençant à manquer dans les greniers, les activités de cueillette et de prédation sur le milieu s'intensifient.

La cinquième lunaison est nommée ñe kene kanna, 1itt. "je laisse bouche". Ce qui signifie qu'il n'y a plus rien à manger, plus rien à porter à la bouche.

La sixième teder na acigbó (manger dans la poignée). C'est le mois des récoltes et de 1 'abondance retrouvée.

La septième tetejan (partager, disperser). Selon certains informateurs, cette appellation fait réfërence au partage de la récolte entre ceux qui ont trayaillê sur le même champ. Selon d'autres, elle éyoque la dispersion des villageois qui ont travaillé sur un même lieu. Sur l'ile de Bubaque, les champs peuvent être très éloignés des villages (parfois on cultive même sur une autre $\hat{\imath} l e$ ). Pendant la saison culturale, les. Bijogo vivent dans des habitations provisoires édifiées à la lisière des champs. Après la récolte, ils"se dispersent" et regagnent leurs villages respectifs.

On remarquera que les critères de nomination des lunaisons ne relèvent pas d'un même champ conceptuel. Les mois sont nommés soit en fonction du climat, soit d'un ordre : celui qui vient après tel autre, sott d'un état de pénurie ou d'abondance, soit d'une tâche. Très peu de personnes connaissent les noms de toutes les lunaisons et parmi celles qui possèdent ce sayoir, très peu voulaient répondre quand je demandais. en quelle lunaison nous nous trouvions. A cela plusieurs raisons. Outre. le fait qu'un Bijogo s'offusque toujours d'une question trop directe, on ne comprenait pas que je m'intéresse à ces questions puisqu'on savait que je disposais de la connaissance du calendrier "officiel". On me demandait d'ailleurs souvent en créole le mois et le quantième. D'autre part, le calendrier lunaire bijogo n'a pas le caractère rigide d'un système absolu mais possède la fluidité des systèmes relatifs. J'ai effectué mon enquête au mois de juin, les premières pluies étaient tombées lors de la lunaison précédente, $j$ 'en ayais donc déduit que nous étions dans la seconde lunaison nommée. Mais les pluies avaient êté peu abondantes et alors qu'en temps normal les semailles auraient dû se faire, personne ne semait. Il était à prévoir que l'on ne sarclerait pas pendant la troisième lunaison qui s'appelle pourtant "le temps de sarcler". L'indécision de mes interlocuteurs reflétait-elle l'indécision du climat ?

Le repérage dans le temps est fondé sur un ensemble de considérations écologiques plutôt que d'observations astronomiques et obẻit à une logique pratique plutôt qu'à une logique théorique. On dira que $I^{\prime}$ on est dans la lunaison eran kadubi (le temps de sarcler) au moment où 1 'on sarcle effectivement et non pas parce que 1 'on est dans la troisième lunaison après la première pluie. On peut penser que ce fait autorise une singularisation régionale mais aussi individuelle. S'il est probable que pour une région donnée tout le monde sarclera en même temps, la période de pénurie peut se produire plus ou moins tôt selon les personnes. Que quelqu'un ait dépensé son riz pour sacrifier à quelque obligation rituelle ou qu'il ait nourri une classe d. âge pour aider 
à la construction d'une maison, ou qu'il se soit simplement montré imprévoyant ou malchanceux, il vivra sa période ñe kene kanna ("je laisse houche") avant les autres et plus longtemps (6).

Il est certain que personne n'éprouve le besoin de récapituler les lunaisons, d'en faire le décompte ou de savoir en laquelle on se trouve pour vaquer à ses occupations. Tout au long de mon enquête, $j^{\prime}$ 'ai eu 1 'impression de créer 1 'objet sur lequel $j$ 'enquêtais, arrachant le nom d'une Iunaison à l'un, celle d'une autre à un second, vérifiant auprès d'un troisième leur ordre de succession, et fabriquant ainsi un calendrier qui n'existe comme tel dans la tête d'aucun Bijogo. Pas seulement parce qu'ils l'auraient oublié, mais aussi parce que ces schèmes temporels ne s'actualisent qu'à l'état pratique dans des situations particulières.

Les phases de Za lune (7)

Dans une lunaison les Bijogo distinguent ces différentes phases :

1. (etaaku) kopeeki, "faucille",

2. (etaaku) edim bono, "qui grandit",

3. (etaaku) yaawanno, je n'ai pu obtenir le sens littéral de cette expression qui désigne la pleine lune, mais on me fournissait un commentaire de ce type : "cela évoque le fait que la pleine lune éclaire corme le soleil levant",

4. (etaaku) yakpeo, "qui meurt",

5. (etaaku) eimpir, pas plus que pour la phase 3 je ne connais le sens littêral de cette expression qui désigne le dernier croissant de la lune; le commentaire était "la lune envoie déjà de 1'obscuritê".

Certaines cérémonies ne sont célébrées qu'à des périodes particulières du cycle lunaire. Il s'agit d'un fait de tradition qui ne demande pas qu'on recoure à une divination pour déterminer le moment favorable.

La religion bijogo consiste en cultes rendus à des puissances fixêes dans des objets. Il existe différentes sortes d'objets qui se distinguent tant par leur forme -- il peut s'agir de sculptures anthropomorphes ou abstraites, de cuvettes de boue, de cornes, de sachets d'herbes, etc. -- que par les pouvoirs qu'ils possèdent. Ces objets nommés orebok se présentent sous forme de collection hiérarchisée disposée sur un autel de terre dans les maisons particulières et dans le sanctuaire du village. L'esprit supérieur du sanctuaire de village est nommé orebok unikan, "esprit grand".

Les sacrifices et libations adressés aux orebok des maisons se font pendant la Zune croissante. I1 s'agit de cérémonies propitiatoires dont la fréquence est fonction des besoins des particuliers qui y procèdent. Au minimum dans l'année il sera cêlébré đeux cérémonies, l'une avant le défrichage pour demander la protection des cultivateurs (cette activité étant dangereuse : risque de blessures avec les outils ou de morsures de serpents) et 1 'autre pour remercier les esprits apaprès la récolte. En général, au cours de l'année bien d'autres occasions se prësentent de sacrifier aux orebok domestiques : maladie, voyage, etc. Tous les ans, on célèbre un rite de régênération des forces de l'orebok unikan. Au cours de cette cérémonie, à laquelle. assistent tous les villageois, est procédé au changement du linge 
rouge qui drape le pied de la statue anthropomorphe de 1 'orebok unikan et du lien rouge qui ceint sa tête. Ce rite est toujours accompli en saison sèche, en forêt où on transporte l'orebok unikan, et pendant La phase décroissante de la lune.

La façon dont sont đistribués ces deux types de rites suggère qu'une lunaison est moins conçue comme un processus cyclique du type naissance-croissance-maturité-décroissance-mort-naissance, etc., que comme un processus digital où croissance s'oppose à dêcroissance. Opposition qui lors des rites serait mise en jeu dans un rapport d'homologie avec d'autres oppositions, ici, esprit domestique/esprit villageois, village/forêt. Sinon comment comprendre qu'un rite de régénération, de re-création, soit accompli pendant la phase décroissante de la lune ?

Par ailleurs, il nous a été rapporté que tous les rites concernant les enfants nouveaux-nês étaient accomplis dans 1a phase kopeeki (premier croissant) de la lunaison. Par exemple, un enfant dont le cordon ombilical a été mal coupé est plus sensible aux maladies qu'un autre. Le rite visant à le protéger des risques qu'il court aura lieu pendant cette période. De même les rites célêtrés pour la naissance de jumeaux dont 1 'excédent de forces est menaçant pour eux et leur mère sont célébrés pendant la période kopeeki.

Si 1 'hypothèse que nous venons de formuler a quelque valeur, ces rites concernant les nouveaux-nés devraient s'opposer à d'autres (funéraires ?) accomplis pendant la phase eimpir (dernier croissant) de la lune. L'état actuel de nos informations ne nous permet pas d'en décider, mais fera 1 'objet d'investigations ultérieures.

Le jour

Le terme yaññ désigne à la fois le temps qui sépare deux levers du soleil et les seules heures éclairées de la journée. On précise pour la journée yañ̃ni imajono (jour que je vois) et pour la nuit yaññ edoncikono (jour que je ne peux voir), mais plus généralement on opposera yaññi (jour) à kuna (nuit).

Les périodes de la journée sont nommées : kankanna (1'aurore), kankooya (le matin), kooko (miliel du jour), kubinni (1'après-midi), anna na nindo (milieu de la nuit). Nindo est le nom du dieu créateur; minuit se dit donc littêralement "au dedans de dieu". I1 n'y a pas de termes spécifiques pour lever et coucher du soleil dénommés comme matin et après-midi.

Aujourd'hui se dit ken; les trois jours qui précèdent et les trois jours qui suivent reçoivent des noms particuliers : nt int ine ngan (avant avant hier), nt int ine (avant-hier), neenoj (hier), uraane (demain), ankoogan (aprềs-demain) et ankoogan kan (après après-demain).

Ces quelques notes sur 1e calendrier des Bijogo nous ont amené à faire 1 'hypothèse que le temps est perçu par cette population selon un modèle d'oscillation entre deux pôles opposês. C'est cette hypothèse qu'il nous faudra vérifier en êtudiant attentivement la distribution des rites dans l'année et dans le mois lunaire. 
NOTES

(1) Le travail de terrain a êté mené essentiellement dans les yillages d'Ancadona et de Tcharo au nord de 1'île de Bubaque, principalement auprès de Enkemeg Kumanoke et de Ompaneg Kanre, avec 1'aide de Francisco Antonio Moreira.

(2) Nous utilisons pour la transcription 1'alphabet phonétique de I'I.A.I. sauf pour $n$ noté $\tilde{n}$.

Nous remercions Rosine Santos (CLAD, Dakar) qui nous a aidé à êtablir un système phonétique normalisê du dialecte de Bubaque.

(3) Rapport scientifique 1981-1983 du L.A. 221.

(4) Leach E., "Deux essais concernant la représentation symbolique du temps" in Critique de Z'Anthropologie (trad. française), Paris, PUF, 1968.

(5) Certaines circonstances peuvent faire varier cette périodicité : pénurie alimentaire ou nombre insuffisant de personnes en âge de célébrer le rite.

(6) Cette lunaison est d'ailleurs parmi celles dont le nom est le mieux connu.

(7) Il ne m'a été fourni aucune indication ni sur les moments différents auxquels on peut observer la lune ni sur les divers quartiers du ciel où elle se montre au cours de son cycle. Je ne sais si cela tient au caractère trop superficiel de mon enquête ou à une absence de prise en compte de ces facteurs par les Bijogo. Dans 1'êtat actuel de mes connaissances, il me semble que sont distingués des moments encadrant les périodes de croissance et de décroissance de la lune. 